\title{
Reclassification of seven honey bee symbiont strains as Bombella apis
}

2

3 Eric A. Smith ${ }^{1}$, Kirk E. Anderson ${ }^{2}$, Vanessa Corby-Harris ${ }^{2}$, Quinn S. McFrederick ${ }^{3}$, and Irene L.

4 G. Newton ${ }^{1 *}$

5

$6 \quad{ }^{1}$ Department of Biology, Indiana University, Bloomington, Indiana, USA

$7 \quad{ }^{2}$ Carl Hayden Bee Research Center, USDA-ARS, Tucson, Arizona, USA

$8{ }^{3}$ Department of Entomology, University of California, Riverside, California, USA

9 *Author for Correspondence: Irene L. G. Newton, Department of Biology, Indiana University,

10 Bloomington, Indiana, USA, (812) 855-3883, $\underline{\text { irnewton@ @indiana.edu }}$

\section{Abstract}

13 Honey bees are important pollinators of many major crops and add billions of dollars annually to

14 the US economy through their services. Recent declines in the health of the honey bee have

15 startled researchers and lay people alike as honey bees are agriculture's most important

16 pollinator. One factor that may influence colony health is the microbial community. Although

17 honey bee worker guts have a characteristic community of bee-specific microbes, the honey bee

18 queen digestive tracts are colonized predominantly by a single acetic acid bacterium tentatively

19 named Candidatus Parasaccharibacter apium. This bacterium is related to flower-associated

20 microbes such as Saccharibacter floricola, and initial phylogenetic analyses placed it as sister to

21 these environmental bacteria. We used a combination of phylogenetic and sequence identity

22 methods to better resolve evolutionary relationships among $P$. apium, strains in the genus

23 Saccharibacter, and strains in the closely related genus Bombella. Interestingly, measures of 
24 genome-wide average nucleotide identity and aligned fraction, coupled with phylogenetic

25 placement, indicate that many strains labeled as $P$. apium and Saccharibacter sp. are all the same

26 species as Bombella apis. We propose reclassifying these strains as Bombella apis and outline

27 the data supporting that classification below.

28 Key Words

29 Bacterial classification, honey bee, evolution, phylogenetics, average nucleotide identity

30

31

32

33

34

35

36

37

38

39

40

41

42

43

44

45

46 


\section{Introduction}

The honey bee (Apis mellifera) is extremely important economically because of the

49 pollination services it provides to numerous agricultural crops. As a result, there is increasing

50 interest in determining how the microbiome supports and influences bee function. While a honey

51 bee colony is made up of individuals with distinct roles, or castes, the majority of studies on bee

52 microbiomes have focused on workers. The microbial community of worker bees consists of

53 eight to ten core bacterial species (Anderson et al., 2016; Martinson et al., 2011; 2012; Moran,

54 2015; Moran et al., 2012; Sabree et al., 2012). The characterization of these groups led to

55 speculation about their role in honey bee health and whether or not they provision nutrients

56 (Moran, 2015) or assist in the breakdown of plant-derived carbohydrates, as is the case in other

57 insect-microbe interactions (Douglas, 2013; Gündüz \& Douglas, 2009; McCutcheon \& Moran,

58 2007). There has also been speculation as to the role of the microbiome in resistance to

59 pathogens, as microbial communities have been shown to protect the bumble bee (Bombus

60 terristris) from the parasite Crithidia bombi (Koch \& Schmid-Hempel, 2011). Honey bee-

61 associated microbes interact with each other in diverse ways both in vitro and in vivo, suggesting

62 that they may interact syntrophically within workers (Martinson et al., 2012; Rokop et al., 2015).

63 While these studies focused on honey bee workers are intriguing, it is surprising that only

64 recently was the microbiome of queen bees throughout development characterized (Tarpy et al., 65 2015).

Interestingly, the microbial community associated with queen bees is vastly different than

67 that of workers and comprises a large percentage of acetic acid bacteria, a group of bacteria

68 present only at very small percentages in workers. One of the primary bacteria that differentiate

69 queens from workers was tentatively named Candidatus Parasaccharibacter apium (Corby-Harris 
et al., 2014). This bacterium is in the family Acetobacteraceae and occupies defined niches

71 within the hive, including: queen guts, nurse hypopharyngeal glands, nurse crops, and royal jelly,

72 and is only rarely found in high abundance outside of these areas (Anderson et al., 2013;

73 Vojvodic et al., 2013). Evidence suggests that it might play a role in protecting developing larvae

74 and queens from pathogens such as Nosema (Corby-Harris et al., 2014; 2016). Given that this

75 bacterium makes up a large proportion of the queen gut microbiome, it is possible that it plays an

76 important role in queen nutrition, protection from pathogens, and possibly modulating queen

77 fertility, fecundity, and longevity (Anderson et al., 2018).

78 We sought to determine the evolutionary relationships between strains of P. apium,

79 strains of the closely related genus Saccharibacter, and characterized sequences from strains of

80 the recently named genus Bombella, using previously published data (see Table 1 for information

81 on all genomes used in this study). Using a combination of phylogenetic and sequence similarity

82 methods, we found that many genomes labeled as $P$. apium or Saccharibacter $s p$. are actually the

83 same species as strains of the previously described species Bombella apis. We reclassify these

84 strains and outline the data supporting this reclassification below.

85

\section{Materials and methods}

$87 \quad 16 S$ rRNA phylogeny

To determine relatedness of the strains classified as P. apium and Saccharibacter spp.

89 compared to existing Bombella and Saccharibacter species, we compared 16S rRNA gene

90 sequences of these genomes to one another. We first downloaded all $16 \mathrm{~S}$ sequences from NCBI

91 that were labeled as Bombella, Parasaccharibacter, or Saccharibacter (Table 1). We also

92 included Gluconobacter oxydans $\mathrm{H} 24$ for use as an outgroup. All sequences were aligned using 
93 the SINA aligner (Pruesse et al., 2012); parameters used were set using the --auto option. A

94 maximum likelihood phylogeny was constructed using RAxML with the GTRGAMMA

95 substitution model and 1000 bootstrap replicates (v8.2.11, (Stamatakis, 2006)). The final tree

96 was visualized using FigTree (v1.4.2, http://tree.bio.ed.ac.uk/software/figtree/).

Core ortholog phylogeny

To determine genome-wide phylogenetic relationships between strains, we clustered genes from all genomes in Table 1 into orthologous gene clusters using OrthoMCL (v.2.0.9, (Li

101 et al., 2003)). Amino acid sequences were downloaded from NCBI and clustering was performed

102 using default OrthoMCL parameters. We then extracted single copy orthologs using a custom

103 Perl script (available at:

104 https://github.com/esmith1032/bombella_apis/blob/master/parse_ortho_out.pl). We constructed a 105 phylogeny using concatenated amino acid alignments of all single-copy orthologs. The amino 106 acid sequences were aligned using the MAFFT L-INS-I algorithm (v7.310, (Katoh et al., 2002)),

107 and alignments were then concatenated, and used to construct a maximum likelihood phylogeny

108 using RAxML with substitution model PROTGAMMALGF and 1000 bootstrap replicates

109 (v8.2.11, (Stamatakis, 2006)). The final tree was visualized using FigTree (v1.4.2,

110 http://tree.bio.ed.ac.uk/software/figtree/).

112 Calculation of genomic similarity

113 To determine relatedness and proper species assignment, we calculated genome-wide

114 Average Nucleotide Identity (gANI) and aligned fraction (AF) for each pairwise comparison

115 using ANIcalculator (Varghese et al., 2015). Predicted transcript sequences for each pairwise 
comparison were passed to the software, which output gANI and AF in each direction for the

117 pairwise comparison. As gANI and AF can vary depending on the direction of comparison due to

118 differences in genome lengths, we report results for comparisons in both directions.

\section{Results}

$121 \quad 16 S$ rRNA gene phylogeny

To determine phylogenetic relationships of all genomes labeled Parasaccharibacter,

123 Saccharibacter, and Bombella on NCBI, we constructed a maximum likelihood phylogeny using

124 16S rRNA sequences (Figure 1A). This phylogeny indicates that the 16S sequences from the

125 following genomes are all very closely related and represent a single, distinct clade: Bombella

126 apis (16S sequence accession number: NR_157653.1), Saccharibacter sp. M18 (NCBI genome

127 accession number: GCA_002150105.1), Saccharibacter sp. 3.A.1 (GCA_002150125.1),

128 Saccharibacter sp. AM169 (GCA_0007235565.1), P. apium G7_7_3c (GCA_002079945.1), P.

129 apium A29 (GCA_002917995.1), P. apium B8 (GCA_002917945.1), and P. apium C6

130 (GCA_002917985.1). For simplicity, this clade will hereafter be referred to as the "clade of

131 interest". P. apium AS1 is not included in the clade of interest for reasons outlined below. The

132 relationships between sequences within the clade of interest are difficult to determine - as is

133 evidenced by the low bootstrap support for nodes within the clade - owing to the high degree of

134 similarity between the genomes. However, the phylogeny clearly places these accessions with $B$.

135 apis, and sister to $P$. apium AS1. It is interesting to note that, although the sequences all show

136 similar degrees of divergence from B. intestini and B. apis, bootstrap support is quite high for the

137 separation of $B$. intestini from the clade of interest (Figure 1A). 
Core ortholog phylogeny

We used OrthoMCL (v2.0.9 (Li et al., 2003)) to define clusters of orthologous genes

141 (COGs) using the Parasaccharibacter, Saccharibacter, and Bombella genomes listed in Table 1;

142 Gluconobacter oxydans H24 was used as an outgroup. In total, 2,214 COGs were defined, with

143 an average of 8.8 genes per COG. Of these, 1,259 COGs were present as single copies in every

144 genome in the analysis; these were then used to construct a core ortholog phylogeny (Figure 1B)

145 to better resolve phylogenetic relationships.

146 This core orthology phylogeny largely agrees with our 16S rRNA gene phylogeny with

147 one exception: the relationships of $B$. intestini and $P$. apium AS1 have switched such that $B$.

148 intestini is now sister to the clade of interest. Bootstrap support for the nodes in this tree are

149 much higher than in the $16 \mathrm{~S}$ tree, but are still relatively low within the clade of interest.

150 However, bootstrap support among the nodes making up the main backbone of the tree are all

151 100, indicating very high support for the basal nodes (Figure 1B).

154 Given the discrepancy between nomenclature and the phylogeny (the placement of

155 "Parasaccharibacter" and "Saccharibacter spp." within the Bombella group), and considering

156 the short branch lengths within the clade of interest, we calculated genome-wide Average

157 Nucleotide Identity (gANI) and aligned fraction (AF) to clarify species relationships. The

158 general criteria for two genomes to be considered the same species are AF > 0.6 and gANI >

15996.5 (Varghese et al., 2015). Using these criteria, all genomes within the clade of interest should

160 be considered the same species, and distinct from B. intestini and P. apium AS1 (Figure 2),

161 though part of the Bombella genus. Unfortunately, no genome has been published for B. apis. 
162 However, the high degree of support for placement of the B. apis $16 \mathrm{~S}$ rRNA gene sequence

163 within the clade of interest strongly suggests that the genomes within the clade of interest belong

164 to the same species and are all Bombella apis strains.

\section{Discussion} and calculations of aligned fraction and genome-wide average nucleotide identity to determine

169 relationships among symbionts of honey bees, bumble bees, and environmental bacteria. The 170 phylogenetic data largely agree with each other, and with the AF and gANI delimitations of

171 species. The combination of these data indicate that the genomic accessions GCA_000723565.1,

172 GCA_002079945.1,GCA_002917995.1,GCA_002917945.1,GCA_002917985.1,

173 GCA_002150105.1, and GCA_002150125.1 are all the same species, despite being named in

174 NCBI as various strains of Parasaccharibacter apium and Saccharibacter sp. Furthermore, 16S

175 rRNA sequence data indicates that these genomes are all very closely related to Bombella apis

176 and are likely the same species. Given that $P$. apium has been effectively but not validly

177 described (i.e. in accordance with IJSEM standards)(Corby-Harris et al., 2016), while B. apis has

178 been validly described (Yun et al., 2017), we propose renaming the above accession numbers to

179 reflect the proper genus and species assignment while maintaining their current strain

180 designations (see Table 1 for full genus, species, and strain designations). We also propose

181 renaming $P$. apium AS1 to Bombella sp. AS1.

182 Given the ever decreasing cost of genome sequencing and ever increasing number of

183 bacterial genomes being published, this study demonstrates the necessity for exercising caution

184 when 1) describing a new species and 2) assigning a new genome a genus/species designation. It 
185 is becoming increasingly clear that $16 \mathrm{~S}$ rRNA gene sequence data on their own, while useful

186 indicators of approximate phylogenetic placement of a bacterial strain, may not be sufficient for

187 accurate assignment of genus and species to a newly sequenced bacterial strain. Genome-wide

188 data, when available, should be used to determine appropriate genus and species assignments

189 (see Chun et al., 2018).

191 Authors and contributors

192 Conceptualization: EAS and ILGN; Formal analysis: EAS; Writing - original draft: EAS;

193 Writing - review and editing: EAS, KEA, VCH, QSM, ILGN

\section{Conflicts of interest}

196 The authors declare that there are no conflicts of interest.

\section{Funding information}

199 EAS is supported by Agriculture and Food Research Initiative - Education and Workforce

200 Development project accession no. 1019114 from the USDA National Institute of Food and

201 Agriculture

202

\section{Acknowledgements}

204 The authors thank Amelia R. I. Lindsey, Delaney L. Miller, and Audrey J. Parish for helpful 205 feedback on drafts of this manuscript. 


\section{References}

209

210

211

212

213

214

215

216

217

218

219

220

221

222

223

224

225

226

227

228

229

230

231

232

233

234

235

236

237

238

239

240

241

242

243

244

245

246

247

248

249

250

251

252

Anderson, K. E., Ricigliano, V. A., Mott, B. M., Copeland, D. C., Floyd, A. S. \& Maes, P. (2018). The queen's gut refines with age: longevity phenotypes in a social insect model. Microbiome 6, 108. BioMed Central.

Anderson, K. E., Rodrigues, P. A. P., Mott, B. M., Maes, P. \& Corby-Harris, V. (2016). Ecological Succession in the Honey Bee Gut: Shift in Lactobacillus Strain Dominance During Early Adult Development. Microb Ecol 71, 1008-1019. Springer US.

Anderson, K. E., Sheehan, T. H., Mott, B. M., Maes, P., Snyder, L., Schwan, M. R., Walton, A., Jones, B. M. \& Corby-Harris, V. (2013). Microbial Ecology of the Hive and Pollination Landscape: Bacterial Associates from Floral Nectar, the Alimentary Tract and Stored Food of Honey Bees (Apis mellifera). PLOS ONE 8 (N. M. Gerardo, Ed.).

Chun, J., Oren, A., Ventosa, A., Christensen, H., Arahal, D. R., da Costa, M. S., Rooney, A. P., Yi, H., Xu, X.-W. \& other authors. (2018). Proposed minimal standards for the use of genome data for the taxonomy of prokaryotes. Int J Syst Evol Microbiol 68, 461-466. Microbiology Society.

Corby-Harris, V., Snyder, L., Meador, C. A. D., Naldo, R., Mott, B. \& Anderson, K. E. (2016). Parasaccharibacter apium, gen. nov., sp nov., Improves Honey Bee ( Hymenoptera: Apidae) Resistance to Nosema. J Econ Entomol 109, 537-543.

Corby-Harris, V., Snyder, L. A., Schwan, M. R., Maes, P., McFrederick, Q. S. \& Anderson, K. E. (2014). Origin and Effect of Alpha 2.2 Acetobacteraceae in Honey Bee Larvae and Description of Parasaccharibacter apium gen. nov., sp nov. Appl Environ Microbiol 80, 7460-7472 (H. L. Drake, Ed.). American Society for Microbiology.

Douglas, A. E. (2013). Microbial Brokers of Insect-Plant Interactions Revisited. J Chem Ecol 39, 952-961. Springer US.

Gündüz, E. A. \& Douglas, A. E. (2009). Symbiotic Bacteria Enable Insect to Use a Nutritionally Inadequate Diet. Proceedings: Biological Sciences 276, 987-991. Royal Society.

Katoh, K., Misawa, K., Kuma, K. I. \& Miyata, T. (2002). MAFFT: a novel method for rapid multiple sequence alignment based on fast Fourier transform. Nucleic Acids Res 30, 30593066. Oxford University Press.

Koch, H. \& Schmid-Hempel, P. (2011). Socially transmitted gut microbiota protect bumble bees against an intestinal parasite. Proc Natl Acad Sci USA 108, 19288-19292. National Academy of Sciences.

Li, L., Stoeckert, C. J. \& Roos, D. S. (2003). OrthoMCL: identification of ortholog groups for eukaryotic genomes. Genome Res 13, 2178-2189. Cold Spring Harbor Lab.

Martinson, V. G., Danforth, B. N., Minckley, R. L., Rueppell, O., Tingek, S. \& Moran, N. A. (2011). A simple and distinctive microbiota associated with honey bees and bumble bees. Mol Ecol 20, 619-628. Wiley/Blackwell (10.1111).

Martinson, V. G., Moy, J. \& Moran, N. A. (2012). Establishment of Characteristic Gut Bacteria during Development of the Honeybee Worker. Appl Environ Microbiol 78, 28302840.

McCutcheon, J. P. \& Moran, N. A. (2007). Parallel genomic evolution and metabolic interdependence in an ancient symbiosis. Proc Natl Acad Sci USA 104, 19392-19397. National Academy of Sciences.

Moran, N. A. (2015). Genomics of the honey bee microbiome. Curr Opin Insect Sci 10, 22-28. 
Moran, N. A., Hansen, A. K., Powell, J. E. \& Sabree, Z. L. (2012). Distinctive Gut Microbiota of Honey Bees Assessed Using Deep Sampling from Individual Worker Bees. PLoS ONE 7 (G. Smagghe, Ed.). Public Library of Science.

Pruesse, E., Peplies, J. \& Gloeckner, F. O. (2012). SINA: Accurate high-throughput multiple sequence alignment of ribosomal RNA genes. Bioinformatics 28, 1823-1829.

Rokop, Z. P., Horton, M. A. \& Newton, I. L. G. (2015). Interactions between Cooccurring Lactic Acid Bacteria in Honey Bee Hives. Appl Environ Microbiol 81, 7261-7270 (H. Goodrich-Blair, Ed.). American Society for Microbiology.

Sabree, Z. L., Hansen, A. K. \& Moran, N. A. (2012). Independent Studies Using Deep Sequencing Resolve the Same Set of Core Bacterial Species Dominating Gut Communities of Honey Bees. PLoS ONE 7 (S. Bertilsson, Ed.).

Stamatakis, A. (2006). RAxML-VI-HPC: maximum likelihood-based phylogenetic analyses with thousands of taxa and mixed models. Bioinformatics 22, 2688-2690. Oxford University Press.

Tarpy, D. R., Mattila, H. R. \& Newton, I. L. G. (2015). Development of the Honey Bee Gut Microbiome throughout the Queen-Rearing Process. Appl Environ Microbiol 81, 3182-3191 (P. D. Schloss, Ed.). American Society for Microbiology.

Varghese, N. J., Mukherjee, S., Ivanova, N., Konstantinidis, K. T., Mavrommatis, K., Kyrpides, N. C. \& Pati, A. (2015). Microbial species delineation using whole genome sequences. Nucleic Acids Res 43, 6761-6771.

Vojvodic, S., Rehan, S. M. \& Anderson, K. E. (2013). Microbial Gut Diversity of Africanized and European Honey Bee Larval Instars. PLoS ONE 8 (G. Smagghe, Ed.).

Yun, J.-H., Lee, J.-Y., Hyun, D.-W., Jung, M.-J. \& Bae, J.-W. (2017). Bombella apis sp nov., an acetic acid bacterium isolated from the midgut of a honey bee. Int J Syst Evol Microbiol 67, 2184-2188. Microbiology Society. 
287 Figure 1. Maximum likelihood 16S rRNA gene phylogeny (A) and core ortholog phylogeny (B).

288 Accession numbers are given with current names in parentheses. Numbers at nodes represent

289 bootstrap support from 1000 bootstrap pseudoreplicates. The "clade of interest" is marked by a

290 purple dot.

291 Figure 2. Pairwise aligned fraction (A) and genome-wide average nucleotide identity (B) of all

292 strains used in this study. Accession numbers are given with current names in parentheses.

293 Colors in each cell scale with the metric. Aligned fraction scales from 0-100, while gANI scales

294 from 0-1. To be considered the same species, two genomes should have aligned fraction $>0.6$

295 and gANI > 96.5 (Varghese et al., 2015). Both metrics can vary depending on which genome is

296 considered the reference for alignment, and we made the calculations in both directions.

297 Genomes listed on the horizontal axis were considered as the reference for these calculations. 
306 Tables

307 Table 1. GenBank accession number, current name, isolation source, genome size, \% GC, 308 and proposed new names of the strains used in this study.

\begin{tabular}{|c|c|c|c|c|c|}
\hline GenBank accession & Current name & Isolation source $^{1}$ & Genome size (Mbp) & $\% \mathrm{GC}$ & Proposed new name \\
\hline 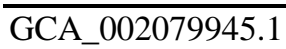 & Parasaccharibacter apium G7_7_3c & Apis mellifera hindgut $^{\mathrm{a}}$ & 2.01 & 59.42 & Bombella apis G7_7_ \\
\hline GCA_002917995.1 & Parasaccharibacter apium A $2 \overline{9}$ & Apis mellifera larva ${ }^{\mathrm{a}}$ & 2.01 & 59.39 & Bombella apis A 29 \\
\hline GCA_002917945.1 & Parasaccharibacter apium B8 & Apis mellifera larva ${ }^{\mathrm{a}}$ & 2.01 & 59.38 & Bombella apis $\mathrm{B} 8$ \\
\hline GCA_002917985.1 & Parasaccharibacter apium C6 & Apis mellifera larva ${ }^{\mathrm{a}}$ & 2.01 & 59.38 & Bombella apis $\mathrm{C} 6$ \\
\hline GCA_000723565.1 & Saccharibacter sp. AM169 & Apis mellifera stomach $^{\mathrm{b}}$ & 1.98 & 59.32 & Bombella apis AM16 \\
\hline GCA_002150105.1 & Saccharibacter sp. M18 & Apis mellifera stomach $^{\mathrm{c}}$ & 2.01 & 59.35 & Bombella apis M18 \\
\hline GCA_002150125.1 & Saccharibacter sp. 3.A.1 & Honey $^{\mathrm{c}}$ & 2.01 & 59.41 & Bombella apis 3.A.1 \\
\hline GCA_002592045.1 & Parasaccharibacter apium AS1 & Apis melliera larva & 1.85 & 52.64 & Bombella sp. AS1 \\
\hline GCA_002003665.1 & Bombella intestini $\mathrm{R}-52487$ & Bombus lapidarius crop $^{\mathrm{d}}$ & 2.02 & 54.94 & N/A \\
\hline GCA_000378165.1 & Saccharibacter floricola DSM15669 & Flower ${ }^{e}$ & 2.38 & 51.22 & N/A \\
\hline GCA_000311765.1 & Gluconobacter oxydans $\mathrm{H} 24$ & Industrial sample ${ }^{\mathrm{f}}$ & 3.82 & 56.24 & N/A \\
\hline
\end{tabular}

$310{ }^{1}$ Isoloation source references: ${ }^{\mathrm{a} C o r b y-H a r r i s ~ \& ~ A n d e r s o n ~ 2018, ~}{ }^{\mathrm{b}}$ Chouaia et al. 2014, ${ }^{\mathrm{c}}$ Veress et 311 al. 2017, ${ }^{\mathrm{d}} \mathrm{Li}$ et al. 2016, ${ }^{\mathrm{e}} \mathrm{Jojima}$ et al. 2004, ${ }^{\mathrm{f}}$ Ge et al. 2013 
$42 \quad$ GCA_002079945.1 (Parasaccharibacter apium G7_7_3c)

_._._._._...... GCA_002150125.1 (Saccharibacter sp. 3.A.1)

GCA_002150125.1 (Saccharibacter sp. 3.A.1)

GCA_000723565.1 (Saccharibacter sp. AM169)

GCA_002917945.1 (Parasaccharibacter apium B8)

GCA_002917985.1 (Parasaccharibacter apium C6)

GCA_002917995.1 (Parasaccharibacter apium A29)

GCA_002592045.1 (Parasaccharibacter apium AS1)

GCA_002003665.1 (Bombella intestini R-52487)

GCA_00378165.1 (Saccharibacter floricola DSM15669)

GCA_000311765.1 (Gluconobacter oxydans H24)

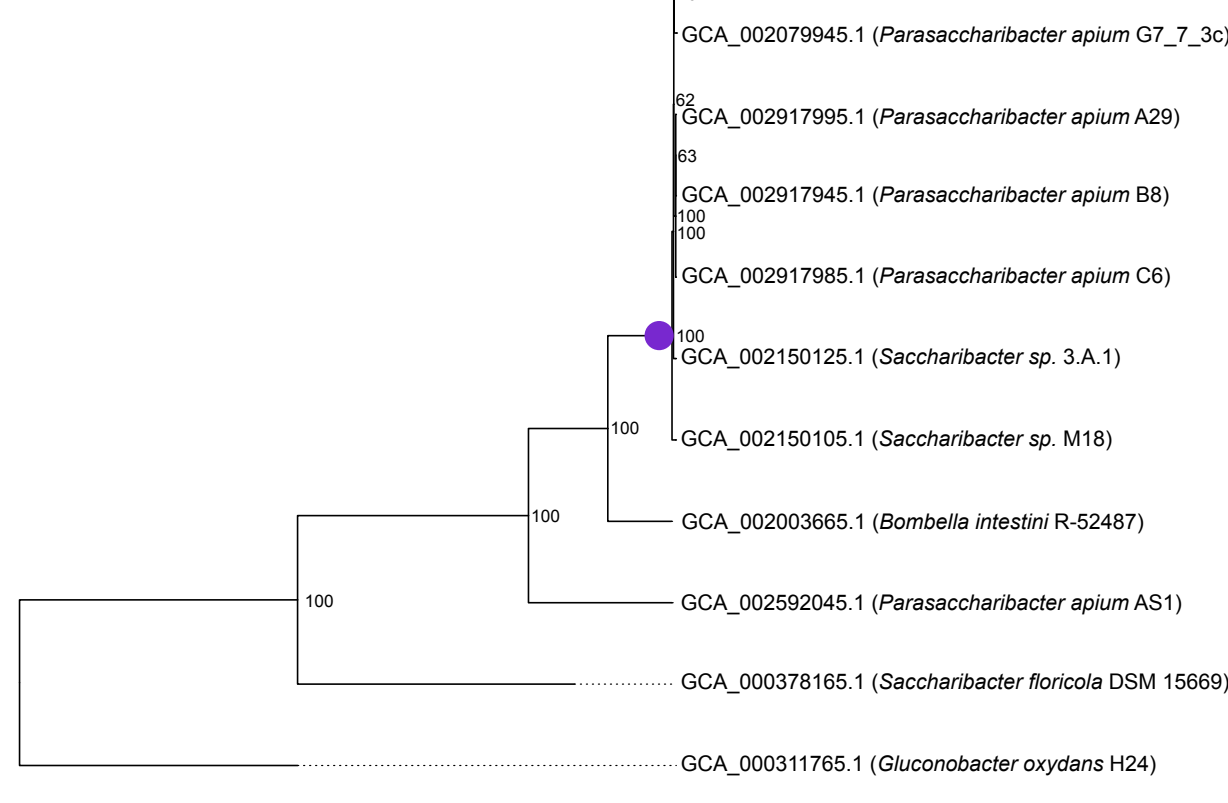


Aligned Fraction (AF)

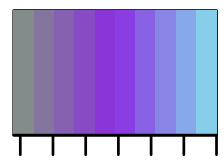

$\begin{array}{lll}0.4 & 0.7 & 1\end{array}$ $\mathrm{AF}$

B

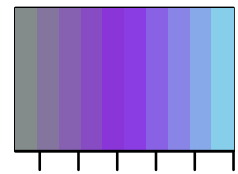

$\begin{array}{lll}75 & 85 & 95\end{array}$ gANI

\begin{tabular}{|c|c|c|c|c|c|c|c|c|c|c|}
\hline 1 & 0.96 & 0.96 & 0.96 & 0.96 & 0.96 & 0.96 & 0.91 & 0.71 & 0.46 & GCA_000723565.1 (Saccharibacter sp. AM169) \\
\hline 0.95 & 1 & 0.95 & 0.95 & 0.95 & 0.95 & 0.95 & 0.89 & 0.7 & 0.45 & GCA_002079945.1 (Parasaccharibacter apium G7_7_3c) \\
\hline 0.95 & 0.95 & 1 & 0.99 & 0.99 & 0.95 & 0.95 & 0.89 & 0.7 & 0.44 & GCA_002917995.1 (Parasaccharibacter apium A29) \\
\hline 0.94 & 0.95 & 0.99 & 1 & 0.99 & 0.95 & 0.95 & 0.89 & 0.7 & 0.44 & GCA_002917945.1 (Parasaccharibacter apium B8) \\
\hline 0.94 & 0.95 & 0.99 & 0.99 & 1 & 0.94 & 0.95 & 0.89 & 0.7 & 0.44 & GCA_002917985.1 (Parasaccharibacter apium C6) \\
\hline 0.92 & 0.92 & 0.92 & 0.92 & 0.92 & 1 & 0.94 & 0.89 & 0.68 & 0.45 & GCA_002150105.1 (Saccharibacter sp. M18) \\
\hline 0.95 & 0.95 & 0.95 & 0.95 & 0.95 & 0.96 & 1 & 0.9 & 0.7 & 0.46 & GCA_002150125.1 (Saccharibacter sp. 3.A.1) \\
\hline 0.89 & 0.89 & 0.89 & 0.89 & 0.89 & 0.91 & 0.89 & 1 & 0.71 & 0.49 & GCA_002003665.1 (Bombella intestini R-52487) \\
\hline 0.75 & 0.75 & 0.75 & 0.75 & 0.75 & 0.75 & 0.75 & 0.77 & 1 & 0.54 & GCA_002592045.1 (Parasaccharibacter apium AS1) \\
\hline 0.38 & 0.38 & 0.38 & 0.38 & 0.38 & 0.39 & 0.39 & 0.42 & 0.43 & 1 & GCA_000378165.1 (Saccharibacter floricola DSM15669) \\
\hline 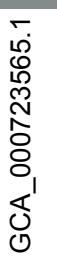 & 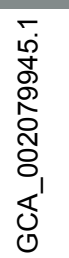 & 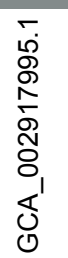 & 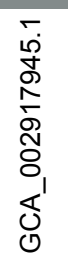 & 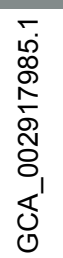 & 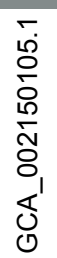 & 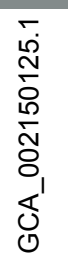 & 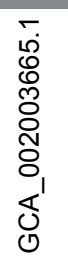 & 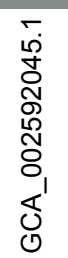 & 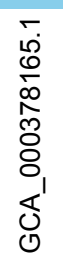 & \\
\hline
\end{tabular}

\section{gANI}

\begin{tabular}{|c|c|c|c|c|c|c|c|c|c|c|}
\hline 100 & 99.24 & 99.27 & 99.27 & 99.27 & 98.82 & 99.22 & 82.96 & 76.03 & 71.91 & GCA_000723565.1 (Saccharibacter sp. AM169) \\
\hline 99.24 & 100 & 99.41 & 99.41 & 99.41 & 98.89 & 99.34 & 82.97 & 75.98 & 71.91 & GCA_002079945.1 (Parasaccharibacter apium G7_7_3c) \\
\hline 99.27 & 99.41 & 100 & 100 & 100 & 98.93 & 99.37 & 82.96 & 76.02 & 71.89 & GCA_002917995.1 (Parasaccharibacter apium A29) \\
\hline 99.27 & 99.41 & 100 & 100 & 100 & 98.93 & 99.37 & 82.96 & 76.02 & 71.89 & GCA_002917945.1 (Parasaccharibacter apium B8) \\
\hline 99.27 & 99.41 & 100 & 100 & 100 & 98.93 & 99.37 & 82.97 & 76.02 & 71.89 & GCA_002917985.1 (Parasaccharibacter apium C6) \\
\hline 98.82 & 98.89 & 98.93 & 98.93 & 98.93 & 100 & 98.86 & 83.07 & 76.06 & 71.85 & GCA_002150105.1 (Saccharibacter sp. M18) \\
\hline 99.22 & 99.34 & 99.37 & 99.37 & 99.37 & 98.86 & 100 & 83.02 & 76 & 71.87 & GCA_002150125.1 (Saccharibacter sp. 3.A.1) \\
\hline 82.96 & 82.96 & 82.94 & 82.94 & 82.95 & 83.07 & 83 & 100 & 76.39 & 71.88 & GCA_002003665.1 (Bombella intestini R-52487) \\
\hline 76.03 & 75.99 & 76.02 & 76.02 & 76.02 & 76.06 & 76.01 & 76.38 & 100 & 71.94 & GCA_002592045.1 (Parasaccharibacter apium AS1) \\
\hline 71.92 & 71.9 & 71.89 & 71.89 & 71.89 & 71.85 & 71.86 & 71.88 & 71.95 & 100 & GCA_000378165.1 (Saccharibacter floricola DSM15669) \\
\hline 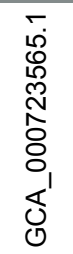 & 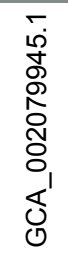 & 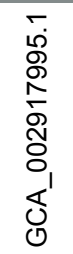 & 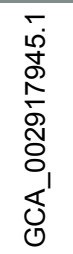 & 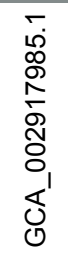 & 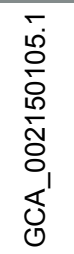 & 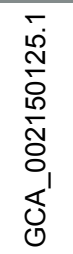 & 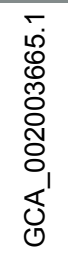 & 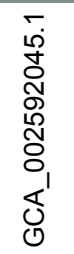 & 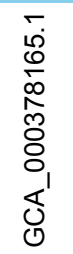 & \\
\hline
\end{tabular}

